\title{
Environmental isomers and continuum models in liquids with emphasis on water and benzene
}

\author{
Ernest Grunwald and Colin Steel \\ Brandeis University, Waltham, MA 02254, USA.
}

\begin{abstract}
Environmental isomers are molecules of the same molecular species which are distinguishable because they exist in different liquid cages. This paper derives the conditions for distinguishability when the cages exist in dynamic equilibrium. The approach is similar to that used in treating the coalescence of spectral lines in dynamic magnetic resonance. For both water and benzene there is evidence that the liquid might be a mixture of two main environmental isomers. By our criteria the suggested isomers for water would be casily distinguishable at ordinary $\mathrm{T}$ and $\mathrm{P}$, while those for benzene would not be.
\end{abstract}

\section{INTRODUCTION}

The cage effect in liquids permits the existence of environmental isomers, which are defined as molecules that belong to the same molecular species but are distinguishable because they exist in different liquid cages. In this paper we shall derive the conditions for distinguishability. It will turn out that distinguishability depends both on differences in the interaction energy of a molecule with its various cages, and on the cage lifetimes.

For example, the pair potential of two benzene molecules, according to computer simulations, has two stable minima: a tilted-T configuration $(\mathrm{t} t)$ at $-9.7 \mathrm{~kJ}$, and a displaced-parallel configuration $(\mathrm{p})$ at $-8.8 \mathrm{~kJ}$, as shown in Fig. 1 (ref. 1). If this is accepted, then the benzene molecules in liquid benzene can be divided into three groups, depending on whether the ring neighbors are $|t| t|| t \mid d$, or Idld. In fact, there is no compelling, evidence that liquid benzene is a mixture of isomers, and we shall find that the computed energy differences are too small for operational distinguishability.
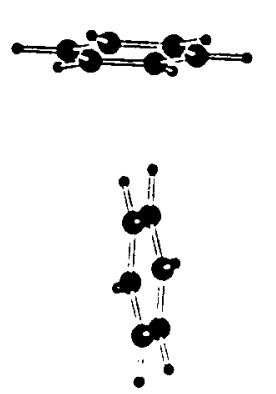

(a)
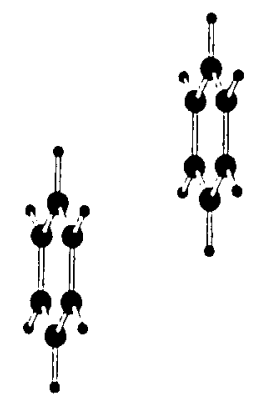

(b)

On the other hand, for liquid water there is thermodynamic, dielectric and spectroscopic evidence (refs. 2-8) that the liquid may be an equilibrium mixture of two "states", but broad acceptance of the two-state model has been prevented by the lack of a plausible description of the nature of the states. 
TABLE 1. Fitted properties and proposed cage structures for the two states of water (refs. 3-7).

\begin{tabular}{ccc}
\hline Cage environment & $1 \mathbf{4 w}$ & $15 \mathbf{w}$ \\
\hline molecules in cage wall & 4 & 5 \\
configuration & ice-like & $\begin{array}{l}\text { one side ice-like, other } \\
\text { side net } \begin{array}{c}\text { non-polar } \\
0.31\end{array}\end{array}$ \\
fractional amount $(298 \mathrm{~K})$ & 0.69 & $0.91 \mu_{\mathrm{W}}$ \\
resultant cage dipole & $2.01 \mu_{\mathrm{W}}$ & $10.5 \mathrm{~kJ} / \mathrm{mol}$ \\
$\Delta \mathrm{H}_{4 \rightarrow 5}$ & & $24 \mathrm{~J} / \mathrm{mol} \cdot \mathrm{K}$ \\
$\Delta \mathrm{S}_{4 \rightarrow 5}$ & & ca. $1.5 \times 10^{11} \mathrm{~s}^{-1}$ \\
$1 / \tau$ & &
\end{tabular}

The potential-energy surface of $\mathrm{HOH}$ is well-known and does not support a case for structural or conformational isomers. And if the states were distinct hydrogen-bonded complexes, one would expect to find many states to be significant, rather than merely two. Grunwald has argued that the states are environmental isomers (ref, 7), and we shall find that the fitting parameters which describe the properties of the two states indeed satisfy conditions for distinguishable environmental isomers. Some of these fitted properties are given in Table 1.

\section{EXCHANGE AVERAGING IN THE TIME AND FREQUENCY DOMAINS}

Liquid cages interconvert. For instance, the conversion of $|t| t$ to $|t| d$ benzene requires no more than a near $90^{\circ}$ rotation and minor translation of a benzene molecule in the surrounding cage, a process whose mean time at room temperature might be of the order of $10^{-11} \mathrm{~s}$. Because of the interconversion of liquid cages, we shall find that conditions for distinguishability of environmental isomers resemble those for exchange averaging of NMR spectral lines, and we shall therefore begin with a review of chemical exchange in NMR (refs. 9-12). Our conclusion will be that exchangeaveraging is not a peculiarly magnetic-resonance phenomenon: It is a general symptom of frequency-switching in waves. The magnetic field serves only to separate the nuclear-spin energy levels.

Fig. 2a shows the well-known sequence of NMR absorption line shapes with increasing rate of exchange for two equally populated chemical shifts. The initially discrete spectral lines first broaden, then coalesce, and then sharpen up as a single line. These spectra were calculated from the McConnell-Bloch equations (ref. 11), assuming slow passage, negligible $T_{2}$ relaxation and negligible If saturation.

We shall now show that an identical pattern of line broadening and coalescence is obtained for any "wave switching". Thus Fig. 3 shows two waves of equal amplitude and arbitrary physical nature, switching back and forth between two frequencies $v_{\Lambda}$ and $v_{13}$. The frequency-switches must be sudden, so that wave-motion during the transit-times is negligible. Let $\tau_{\Lambda}$ and $\tau_{\mathrm{B}}$ denote the mean residence times at $\nu_{A}$ and $v_{B 3}$ and let $\tau$ be defined by $1 / \tau=1 / \tau_{A}+1 / \tau_{B 3}$. The individual residence times at $v_{\Lambda}$ and $v_{B}$, between frequency switches, of course vary stochastically about their mean values according to an exponential distribution of residence times, as required by first-order kinetics The wave trains used in our calculations encompass $10^{4}$ to $10^{5}$ frequency switches, with exponential distributions of waiting times. 


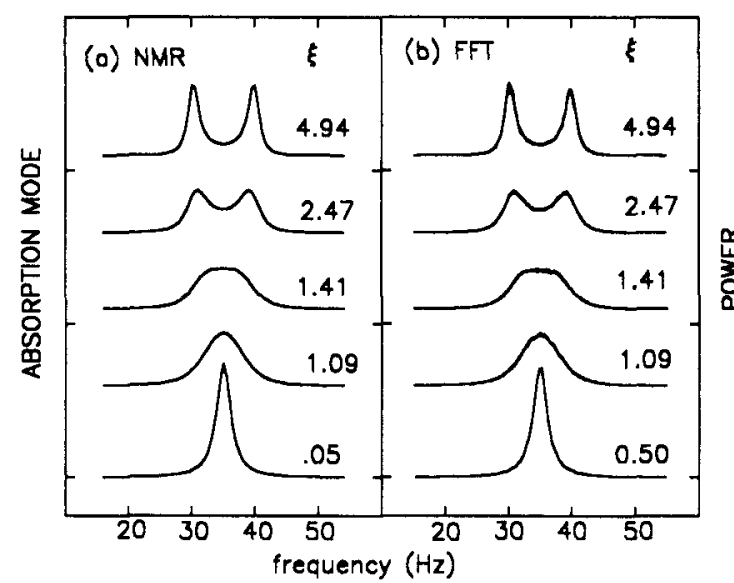

Fig. 2.(a) Magnetic resonance absorption spectra as a function of distinguishability index $(\xi)$. Curves calculated from McConnell-Bloch equations.

(b) Normalized power spectra resulting from stochastic FFT under corresponding conditions. Each compartment is an overlay of FFT's resulting from a frequency-switching wave train and an energy switching state function (ref 18).

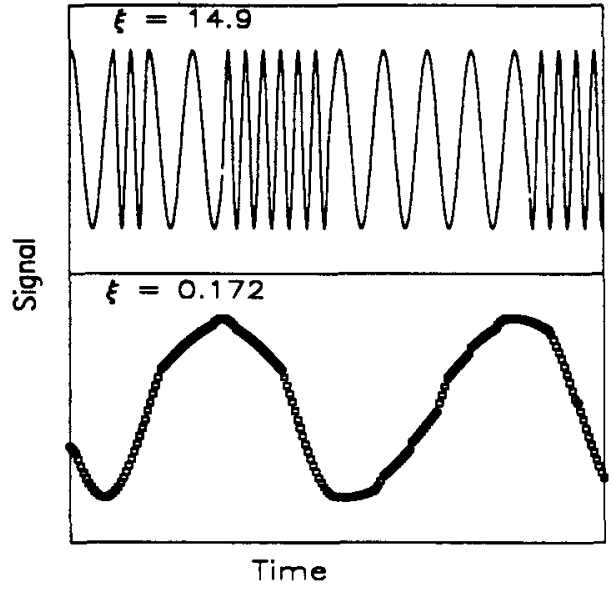

Fig. 3. Typical waveforms subjected to stochastic FFT. The upper wavetrain yields a power spectrum consisting of two well resolved lines. The lower wavetrain yields a single collapsed line.

Fig. $2 b$ shows the power spectra obtained from discrete Fast-Fourier transforms (FFT's) of such wave-trains into the frequency domain. The $v$ 's and $\tau$ 's match those of Fig. 2a. It is clear that the FFT-power spectra and the NMR absorptions show essentially identical shapes.

The technique of formulating the problem of frequency switching first in the time domain and then transforming it into the frequency domain provides a comfortable link to the formulations of chemical kinetics. It also permits certain properties of the spectra to be derived from established Fourier transform theorems (refs. 13,14). For spectral coalescence it can thus be shown that the absolute values of $v_{A}$ and $v_{B}$ are arbitrary, because coalescence depends only on their difference $\left|v_{\Lambda}-v_{13}\right|$ as well as the mean time $\tau$ for exchange, while $\left|v_{A}+v_{B}\right| / 2$ determines the centroid of the active spectral range. From the view-point of distinguishability, the relevant parameter is the distinguishability index $\xi$, defined in $\mathrm{Eq} .1$.

$$
\xi=2 \pi\left|v_{\mathrm{B}}-v_{\Lambda}\right| \tau
$$

As shown in Figs. 2, when $\xi \gg 1$ the lines are well resolved. When $\xi \ll 1$, the FFT spectrum consists of a single sharp line. Coalescence occurs in the narrow range $1 \leq \xi \leq \sqrt{2}$. In Fig. 2, where $\tau_{\wedge}=\tau_{13}$ and the wave-amplitudes are equal, the coalescence point is at $\xi=\sqrt{2}$.

\section{DISTINGUISHABILITY INDEX AND ENVIRONMENTAL ISOMERISM}

Although the translation and rotation (TR) of caged molecules are hindered motions (librations) and the associated energy levels thus vary with the nature of the cage, the spacing of the TR levels is dense enough so that the energy per mode is close to $\mathrm{kT}$, the classical limit for an oscillator. In the following, we shall therefore track the electronic energy and the electronic wave function of the caged molecule. In particular, we shall assume that there are just two kinds of cages, that the nature of the cage surrounding a given molecule interconverts between one kind and the other, and that the 
molecule therefore continually switches its environment. We treat the interaction of the molecule with its cage as a perturbation, and assume that the caged molecule remains in its electronic ground state. Within this framework, cage switching is tantamount to switching of the related perturbation energy of the electronic ground state. The problem is simplified because the molecular motions are slow enough so that Ehrenfest's Adiabatic Principle applies (ref. 15). A change in a cage-wall proceeds on the time-scale of molecular motions, while the electronic energy of the electronic ground state responds to the change on the much shorter time-scale of electronic motions. Accordingly, a caged molecule, on cage-switching, arrives in its new cage with the electronic wave function and associated energy essentially in a stationary state. As a further simplification, we shall distinguish between relatively slow, qualitative changes in the "structure" of the cage wall, and stochastic noise due merely to thermal motions of the molecules in the cage wall. The noise fluctuates on the timescale of molecular motions, while qualitative changes in the structure of the cage wall occur on a significantly longer time-scale (ref.16). On this basis, the transit time for cage-switching and the correlation time for thermal noise are both long enough for the Adiabatic Principle to apply, while the same transit time is short compared to the mean lifetime of the cage. Cage-switching may thus be viewed as a sudden transit from one stationary, albeit noisy, wave function to another, in the same sense that spectral line-switching in NMR, in the presence of magnetic thermal noise, is viewed as a sudden event.

In the following, we shall approach the problem of distinguishability of cage environments in two steps. First we shall examine sudden cage-switching in the absence of thermal noise; then we shall include thermal noise. As might be expected, the inclusion of thermal noise is important. For definiteness, let the two cage environments be denoted by la and $l b$. The wave functions for the caged molecule in the two environments is then $\Psi_{\mathrm{a}(\mathrm{b})}(\mathrm{x}, \mathrm{t})=\phi_{\mathrm{a}(\mathrm{b})}(\mathrm{x}) \exp \left(-2 \pi \mathrm{i} \varepsilon_{\mathrm{a}(\mathrm{b})} \mathrm{t} / \mathrm{h}\right)$, where $\varepsilon_{\mathrm{a}}$ and $\varepsilon_{\mathrm{b}}$ are the two state energies as perturbed by the $A$ and $B$ cages. The time-dependent part of the wave function has frequencies $v_{\mathrm{a}(\mathrm{b})}=\varepsilon_{\mathrm{a}(\mathrm{b})}$ /h so that the distinguishability index (1) becomes:

$$
\xi=2 \pi\left|\varepsilon_{\mathrm{b}} / \mathrm{h}-\varepsilon_{\mathrm{a}} / \mathrm{h}\right| \tau=2 \pi\left|\Delta \varepsilon_{\mathrm{ab}} / \mathrm{h}\right| \tau
$$

Absolute values of $\varepsilon_{\mathrm{a}}$ and $\varepsilon_{\mathrm{b}}$ are not needed. Distinguishability of states in cage switching is therefore analogous to distinguishability of spectral lines in spectral-frequency switching.

This conclusion is clearly demonstrated by Fourier transformation. In the examples to be presented, the stochastically exchanging state waves have equal amplitudes and mean lifetimes $\left(\tau_{A}=\tau_{13}\right)$. This result is shown in Fig. $2 b$, in which each compartment shows an overlay of two power spectra with the same value of $\xi$, one for spectral-frequency switching and the other for cage switching. The overlays are practically identical. The differences are consistent with $3 \%$ random errors owing to the finite lengths of the wavetrains. Thus, within this framework, stochastic sudden switching between two stationary quantum states produces the same kind of power spectra as are observed in magnetic resonance or, indeed, in any stochastic frequency switching of a harmonic motion. At high values of $\xi$ the two state-waves are well resolved, at low $\xi$ they are exchange-averaged, and coalescence occurs when $\xi$ is of order 1 to $\sqrt{2}$. In quantum mechanics, state-waves are not observables - one needs to perform an operation to obtain an observable result. We shall therefore state, without proof, that operationally the distinction between two interconverting energy states $\varepsilon_{\mathrm{B}}$ and $\varepsilon_{\mathrm{A}}$ is always a matter of probability. The probability increases with increasing $\xi$ and is greater than $1 / 2$ when $\xi>10$. On this basis, the odds are better than even that molecules in two cages, $l a$ and $l b$, can be distinguished by energy measurements when $\left|\Delta \varepsilon_{\mathrm{ab}}\right|>(10 / 2 \pi) \mathrm{h} / \tau$. 


\section{EFFECT OF THERMAL NOISE}

Even in the absence of cage exchange, the electronic state-energy of the caged molecule fluctuates because of thermal motions of the molecules in the cage wall. Because of limited space we shall give only an outline of our procedure. We assume that the $s$ molecules in the cage wall move independently, and that only one direction of motion, the motion normal to the cage wall, is relevant. We adopt a possible pairwise potential and use it to construct a cage potential for the central molecule such that the potential minimum occurs at the most probable nearest-neighbor distance, which need not coincide with the pairwise minimum but might coincide with the maximum of the pairwise distribution function $g(r)$ for the liquid. Given the cage potential, we calculate the amplitude of the motion in the desired direction when the mean energy of motion, potential + kinetic, equals $k T$. We then use that amplitude and the original pairwise potential to calculate $\sigma_{d}(\varepsilon)$, the standard deviation in the fluctuating perturbation energy for one molecular pair - one molecule in the cage and the other in the cage wall. Finally, the total energy half-width associated with the thermal fluctuations is $\Delta \varepsilon_{\text {them }}=V_{s} \cdot \sigma_{d}(\varepsilon)$. For non-polar liquids the result obtained for $\Delta \varepsilon_{\text {therm }}$ is remarkably robust. For a Lennard-Jones pairwise potential we obtain Eq. 3

$$
\left.\Delta \varepsilon_{\text {therm }}=(4 / 5 \mathrm{~s})^{1 / 2}(\mathrm{kT}) \quad \text { (Lennard-Jones pairwise potential }\right)
$$

The Lennard-Jones parameters $\left(\varepsilon_{\mathrm{LJ}}\right.$ and $\left.\sigma_{\mathrm{IJJ}}\right)$ and the liquid expansion (i.e. the chosen mean nearestneighbor distance) do not enter the final result. Other models we have tried gave results of the same magnitude as (3). We shall therefore adopt Eq. 3 for the energy half-width of benzene cages. Setting the mean nearest-neighbor number $\mathrm{s}=8$, we obtain $\Delta \varepsilon_{\text {thern }}=0.8 \mathrm{~kJ} / \mathrm{mol}$

For water cages we used the dipole-dipole potential tested by Grunwald (ref. 7), and added a Lennard-Jones interaction with $\sigma_{\mathrm{l}, \mathrm{J}}=2.725 \AA$ and $\varepsilon_{\mathrm{L}, \mathrm{J}}=-6.0 \mathrm{~kJ} / \mathrm{mol}$, which together reproduce the observed mean $0-0$ neighbor distance of $2.9 \AA$. Then $\Delta \varepsilon_{\text {therm, } 14 \mathrm{w}}=1.1 \mathrm{~kJ} / \mathrm{mol}$ for the $\mathrm{s}=4$ cage, and $\Delta \varepsilon_{\text {therm, } 15 \mathrm{w}}=1.0 \mathrm{~kJ} / \mathrm{mol}$ for the $\mathrm{s}=5$ cage. These values will be used in the following.

\section{DISTINGUISHABILITY OF NOISY CAGE ENVIRONMENTS}

We begin with Fig. 4, which shows the state energies of benzene molecules in $|a \equiv| t \mid t$ and $|\mathrm{b} \equiv| t \mid \mathrm{p}$ cages. (See Fig. 1.) $\Delta \varepsilon_{\mathrm{ab}}=0.9 \mathrm{~kJ} / \mathrm{mol} ; \Delta \varepsilon_{\mathrm{thern}, \mathrm{al}}=\Delta \varepsilon_{\mathrm{therm}, \mathrm{b}}=0.8 \mathrm{~kJ} / \mathrm{mol}$; the populations of the two states are treated as equal since $\backslash t i p$ has a statistical weight of two. $1 / \tau$ is equated to the rate constant $6 \mathrm{D}_{\text {benz }} / \sigma^{2}$ for the dissociation of a benzene-benzene neighbor pair, where $D_{\text {benz }}$ is the macroscopic coefficient for linear diffusion (ref.16). The value for $1 / \tau$ is $4 \times 10^{10} \mathrm{~s}^{-1}$. Accordingly, $\xi$ $=350$. In Fig. 4 , the sharp lines (a) represent the power spectrum in the absence of thermal noise. Here the states are clearly distinguishable. The broad band (b) shows the same power spectrum with $\Delta \varepsilon_{\text {them }}=0.8 \mathrm{~kJ} / \mathrm{mol}\left(2.0 \times 10^{12} \mathrm{~s}^{-1}\right)$. The states now overlap, and the original resolution is lost.

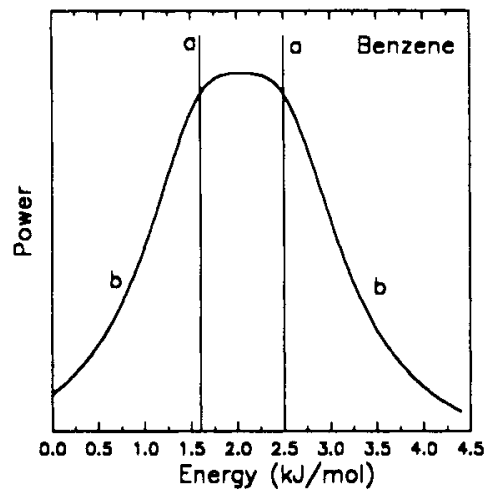

Fig. 4. Simulated power spectrum for benzene molecules in $|t| t$ and $|t| p$ environments, as described in the text. (a) Without thermal noise there are two sharp lines. (b) With thermal noise the two lines broaden and overlap. 


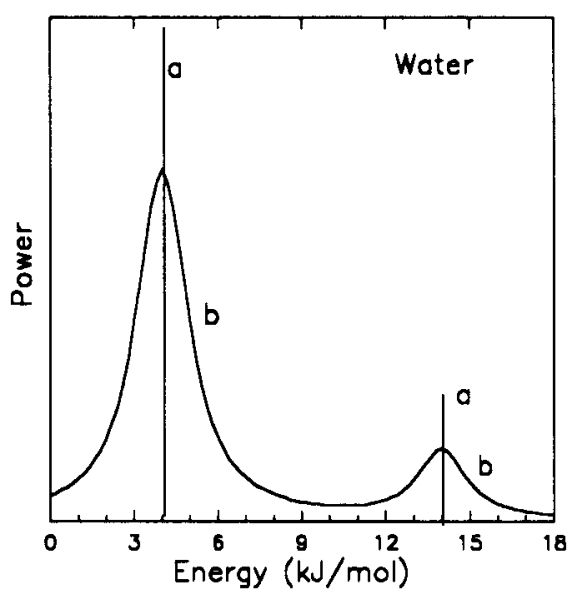

Fig. 5. Simulated power spectrum for water molecules in $14 \mathrm{w}$ and $15 \mathrm{w}$ environments, as described in the text. (a) Without thermal noise there are two sharp lines. (b) With thermal noise the lines broaden but remain well resolved.

Fig. 5 shows the state energies of water molecules in $\backslash a \equiv \backslash 4 \mathrm{w}$ and $\backslash \mathrm{b} \equiv \backslash 5 \mathrm{w}$. (See Table I.) We shall use the following values: fraction of $\backslash 4 \mathrm{w}=0.7, \Delta \varepsilon_{\mathrm{ab}}=10 \mathrm{~kJ} / \mathrm{mol}, \Delta \varepsilon_{\text {therm, } \mathrm{a}}=1.1 \mathrm{~kJ} / \mathrm{mol}$, $\Delta \varepsilon_{\text {therm, } b}=1.0 \mathrm{~kJ} / \mathrm{mol}$, and $1 / \tau=1.5 \times 10^{11} \mathrm{~s}^{-1}$. Accordingly, $\xi=1000$. The sharp lines (a) represent the power spectrum in the absence of thermal noise, while curve (b) shows the broadened power spectrum in the presence of noise. Although broadened, the bands are clearly resolved, and water molecules in these cages are distinguishable environmental isomers. The empirical evidence that liquid water is an 1:1 equilibrium mixture of two "states" therefore need not be discarded for lack of a reasonable structural model. The two states may reasonably be described as environmental isomers.

\section{CONCLUSIONS}

We conclude that there are two conditions for the existence of environmental isomers: (1) $\xi=2 \pi$ $\left|\Delta \varepsilon_{\mathrm{ab}} / \mathrm{h}\right| \tau>$ ca. 10; (2) $\Delta \varepsilon_{\mathrm{ab}}>$ ca. $3 \mathrm{~kJ} / \mathrm{mol}$. Both conditions must be satisfied. When that is not the case, we visualize two possibilities: (1) state-wave overlap when $\xi$ is ca. 0.5 to 5 ; (2) state coalescence when $\xi<0.5$. In case of state-wave overlap the environment of the caged molecule is a quasi-continuum; either a continuum model or a discrete molecular model, as in the Monte Carlo approach (ref. 1), may be used. In case of state coalescence the environment is a genuine continuum, and a continuum model becomes mandatory.

The molecular packing fraction in liquids varies widely with temperature and pressure. Rabinowitch and Wood (ref. 17) have shown that, as the packing fraction increases, the onset of the cage effect is sudden. When the density of the liquid drops below the threshold for cage formation, environmental isomerism disappears. It is therefore conceivable that environmental isomerism is manifest under conditions of relatively high liquid density, but disappears at lower densities. This may be the case for water, because the "anomalies" which justify a two-state model largely disappear as liquid water approaches the critical point. 


\section{REFERENCES}

1. W.L. Jorgensen and D.L. Severance, J. Am. Chem. Soc. 112, 4768-4774 (1990).

2. H.S. Frank, J. Chem. Phys. 13, 493-506 (1945).

3. G.E. Walrafen, J. Chem. Phys. 48, 244-251 (1968). G. E. Walrafen, M. S. Hokmabadi, M. R. Fischer and W.-H. Yang, ibid. $\underline{85}$, 6970-82 (1986)

4. J.D. Worley and I.M. Klotz, J.Chem. Phys. 45, 2868-2871 (1966).

5. C.A. Angell, J Phys. Chem. 75 3698-3705 (1971).

6. S.W. Benson, J. Am. Chem. Soc. 100, 5640-5644 (1978)

7. E. Grunwald, J. Am. Chem. Soc. 108, 5719-5726 (1986).

8. S.W. Benson and E.D. Siebert, J.Am. Chem. Soc. 114, $4269-4276$ (1992).

9. H.S. Gutowsky, D.W. McCall and C.P. Slichter, J. Chem. Phys. 21 279-292 (1953).

10. E. Grunwald, A. Lowenstein and S. Meiboom, J. Chem. Phys. 27 630-640 (1957).

11. H.M. McConnell, J. Chem. Phys. 28, 430-431 (1958).

12. A. Carrington, Introduction to Magnetic Resonance, Chapman and Hall, London, 1979

13. R.N. Bracewell, The Fourier Transform and its Applications, 2nd ed., McGraw-Hill, New York, (1978).

14. D. C. Champeney, Fourier Transforms and their Physical Applications, Academic Press, London, 1973.

15. R.C. Tolman, The Principles of Statistical Mechanics, p. 414, Oxford University Press, London (1938).

16. Our tentative model for the mean time between cage switching is the mean lifetime of an encounter pair.

17. E.Rabinowitch and W.C. Wood, Trans. Farad. Soc. 32, 1381-1387 (1936).

18. In the normalization of the FFT signals, data at negative frequencies and differences in sampling frequencies were managed according to established mathematical theorems. 